\title{
WS5-D02
}

\section{High-resolution Multicomponent Hardrock Seismic Imaging of Mineral Deposits and their Host Rock Structures}

\author{
A. Malehmir* (Uppsala University), S. Wang (Uppsala University), J. \\ Lamminen (Boliden Mineral AB), M. Bastani (Geological Survey of \\ Sweden), C. Juhlin (Uppsala University), K. Vaittinen (Boliden Mineral AB), \\ L. Dynesius (Uppsala University) \& H. Palm (Uppsala University)
}

\section{SUMMARY}

Although applied in the past, there are only a few cases demonstrating the advantages of multicomponent seismic data for mineral exploration. To illustrate this, a test survey using sixty 3C-digital sensors, spaced between 2 to $4 \mathrm{~m}$ and assembled in a $160 \mathrm{~m}$ long landstreamer, was carried out to provide information on shallow structures hosting mineralization and also a magnetic lineament with an unknown origin. The survey, totally about $1.3 \mathrm{~km}$ long, was complemented by Radio MagnetoTelluric (RMT) measurements. Although an explosive source was used to generate the seismic signal, the seismic data show good quality for all the three components. Supported by the RMT results, clear reflections are observed in the horizontal component data at about $25 \mathrm{~m}$ depth, one of them steeply dipping, likely associated with the magnetic lineament. Field static corrections were well estimated thanks to the close shot and receiver spacing and the broadband frequency content of the data. This study demonstrates that multicomponent seismic data can be useful for providing information on shallow structures and linking them to the surface geology. The vertical component data, however, show deeper penetration and better image the crystalline basement and its undulated/faulted surface at about $50 \mathrm{~m}$ depth. 


\section{Amsterdam '14}

\section{Introduction}

Seismic methods are increasingly being used for mineral, geothermal and groundwater exploration. This is evident from a number of case studies recently presented in the literature. Nevertheless, few attempts and studies have been carried out to illustrate the value of multicomponent seismic methods for these purposes. Most mineral deposits, particularly the metallic ones, show significantly larger Swave than P-wave velocity contrast with their host rocks. In addition, S-wave data can provide information about anisotropy and physical properties, useful for both exploration and mine planning. In suitable conditions (e.g., shallow cover), horizontal component data may also provide higher resolution images of the subsurface than traditional vertical component data. This is particularly useful at shallow depths where a great need to link shallow and deeper structures is often desirable to take the advantage of the detailed surface geological observations and shallow boreholes. In this study, we present results from a pilot 3C seismic landstreamer survey carried out in a mineral exploration site in northern Sweden. The current landstreamer configuration, a prototype broadband $(0-800 \mathrm{~Hz})$ based on digital sensors, consists of three segments with twenty $3 \mathrm{C}$-sensors each $2 \mathrm{~m}$ apart and an additional segment with twenty $3 \mathrm{C}$-sensors each $4 \mathrm{~m}$ apart, giving a total streamer length of $200 \mathrm{~m}$. These segments can be towed in parallel or in series, which in combination with synchronized wireless and cabled sensors can address a variety of complex geological problems. The system is especially geared for noisy environments and areas where high-resolution images of the subsurface are needed. It has little sensitivity to electrical noise and measures sensor tilt and applies correction during the acquisition, important in rough terrains and if needed. In this study, structures down to and within a crystalline basement of Precambrian age were targeted. A magnetic lineament was crossed by the profile to test a hypothesis about the origin of the positive magnetic anomaly. It was assumed being either a basement high or a fault in the basement.

\section{Seismic survey}

To generate the seismic signal, 20-40 grams of dynamite fired at about 1.5-0.7 m depth was used. A shot spacing of $10 \mathrm{~m}$ was used and 8 shots were fired before the streamer was moved to a new position ( $80 \mathrm{~m}$ forward). Shots were only fired within the $2 \mathrm{~m}$ spaced sensors; that is the $80 \mathrm{~m}$ tail was (three 3C-segments were used in this study) overlapped. Totally 130 shots were fired, the streamer was moved 16 times and about $1.3 \mathrm{~km}$ of profile was covered within 2.5 days. Seismic data show good quality, likely due to the good coupling between the sleds and the frozen and snowy ground. In addition to the seismic data, RMT data were measured at every $10 \mathrm{~m}$ along a portion of the profile.

\section{Preliminary results}

Vertical component data showed clear first arrivals almost for all the shots and offset ranges. This allowed a good estimation of the refraction static corrections and imaging a clear reflection at about $25 \mathrm{~ms}$, interpreted to be from the crystalline basement. A conventional post-stack processing approach was implemented with a main focus on field statics, retaining high frequencies up to $350 \mathrm{~Hz}$ and velocity analysis. Horizontal component data are strongly contaminated by surface waves. However, after careful processing and high-pass frequency filtering, shallow reflections became visible in some of the processed shot gathers (Figure 1).

Processing results of vertical component data did not show reflections shallower than $20 \mathrm{~ms}$ (about $50-60 \mathrm{~m}$ ) but did indicate an undulated surface where the magnetic lineament was observed (Figure 2). On the other hand, horizontal component data showed a strong sub-horizontal reflection at about $25 \mathrm{~m}$ depth where the unconstrained RMT inversion model also showed a resistivity contrast. In particular, a steeply dipping reflection at the location of the magnetic high lineament was clearly observed in the horizontal (crossline) component data but not in the vertical component data. The vertical component data, however, better imaged the crystalline basement and together with the horizontal component data suggested that the steeply dipping reflection extends into the basement and crosscuts the shallower stratigraphy. Magnetic lineaments and their relationships with the structures controlling the formation of a mineralization has been a matter of discussion in the study area. The 


\section{Amsterdam l'14}

working hypothesis is that the fault zones provide pathways for hydrothermal fluids and basement highs trap these fluids. Thus these structures are interesting from the exploration point of view (pers. comm., N. Saintilan, August 2013). The results of this survey, the strong reflection from the basement and above it, and the steep reflector and its connection to the magnetic lineament, should be verified by diamond drilling to provide a good calibration for the future use of the method in the study area.
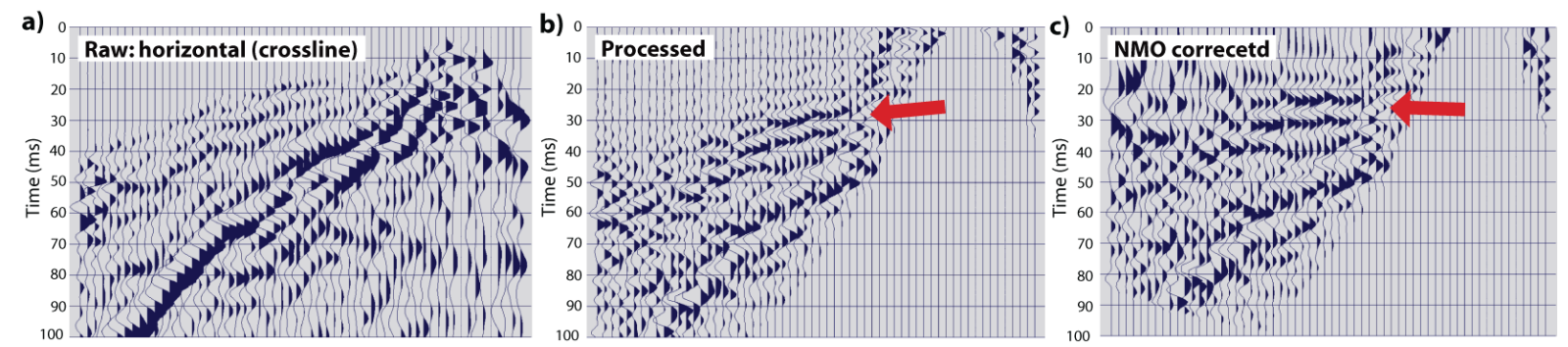

Figure 1 An example horizontal component (crossline) shot gather (a) raw and (b) processed, (c) $N M O$ corrected using a constant NMO velocity of $2750 \mathrm{~m} / \mathrm{s}$. Note the reflections marked by the red arrow at about 20-30 ms.

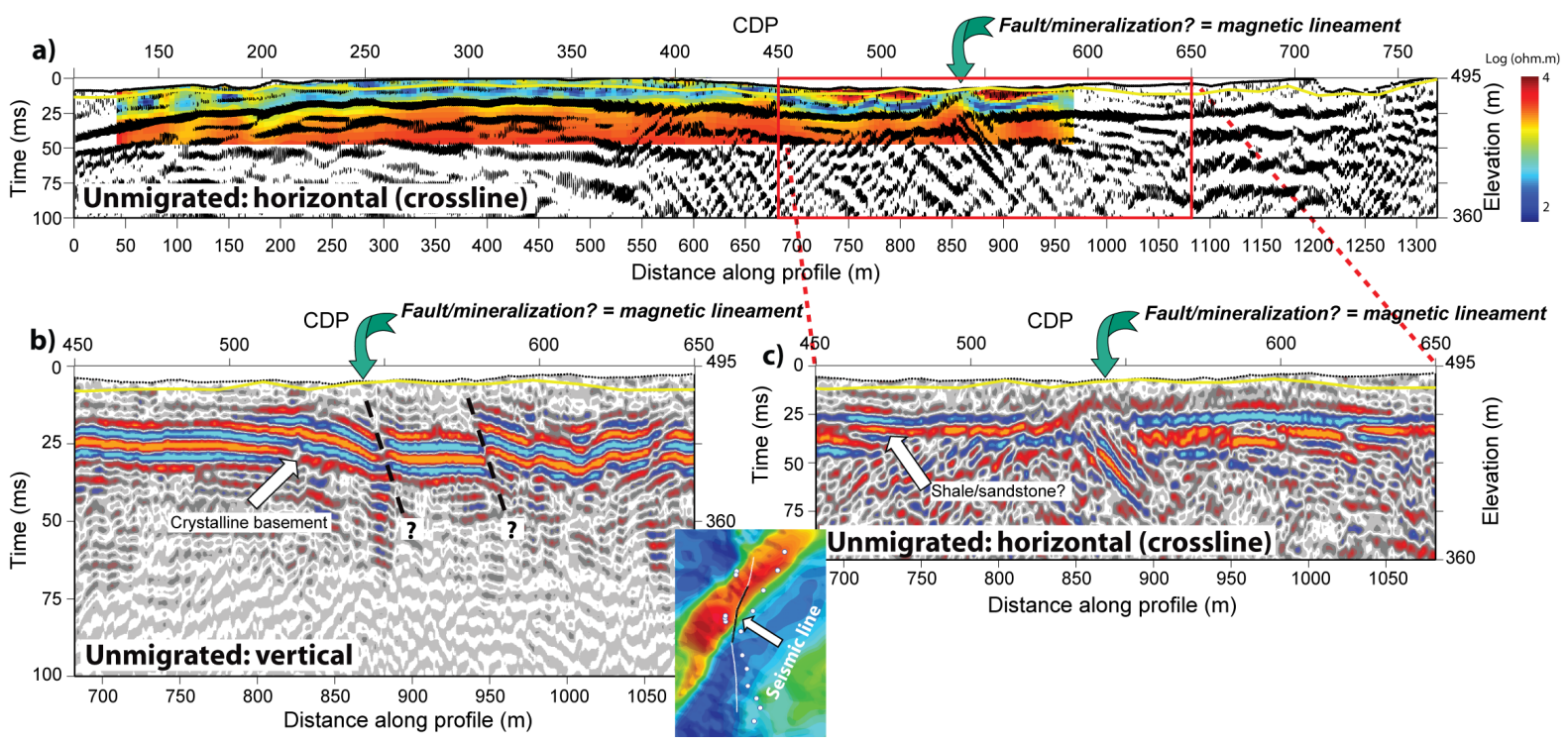

Figure 2 (a) Unmigrated section of horizontal component data superimposed on the RMT results showing an excellent correspondence between the two data sets. A portion of the unmigrated (b) vertical-and (c) horizontal-component data showing the seismic signature of the magnetic lineament in these data. Note that the sub-horizontal reflection in these sections have two different origins and are from two different depths, one likely from the crystalline basement (50-60 $\mathrm{m}$ depth) and another from the contact between shale and sandstone $(25-30 \mathrm{~m}$ depth), respectively. Yellow line on the sections shows the estimated depth to bedrock using first arrivals of the vertical component data.

\section{Conclusions}

The value of using multicomponent seismic data for hardrock seismic imaging is demonstrated using an example from a mineral exploration site. Explosives generate usable horizontal component signals and it is therefore an advantage if these signals are recorded and used by $3 \mathrm{C}$-receivers. The vertical component data show, however, deeper penetrations and successfully image the crystalline basement and its likely faulted structures that maybe related to the origin of the mineralization in the site. Further investigations are required to verify these interpretations.

\section{Acknowledgements}

The seismic survey was carried out within the frame of Trust2.2-GeoInfra (http://trust-geoinfra.se) project sponsored by Formas, BeFo, SBUF, SGU, Boliden, Skanska, FQM, and NGI. 